\title{
Consumption of low doses of fat prevents the postprandial rise in chylomicron particle concentration and remnant accumulation in healthy normolipidaemic males
}

\author{
Anthony P. James ${ }^{1,2 *}$ and John C. Mamo ${ }^{1,2}$ \\ ${ }^{1}$ School of Public Health, Curtin University of Technology, Curtin Health Innovation Research Institute and the Australian Technology Network \\ Universities, Centre for Metabolic Fitness, GPO Box U1987, Perth, WA 6845, Australia \\ ${ }^{2}$ Curtin Health Innovation Research Institute and the Australian Technology Network. Universities, Centre for Metabolic Fitness, Perth, WA 6845, \\ Australia
}

(Received 13 January 2012 - Final revision received 22 May 2012 - Accepted 22 May 2012)

Journal of Nutritional Science (2012), vol. 1, e4, page 1 of 8

doi:10.1017/jns.2012.4

Abstract

Chylomicron particles are continually synthesised and secreted from the intestine even in the absence of ingested fat. It is possible that following consumption of low doses of fat the basal level of chylomicron secretion and subsequent metabolism are sufficient to metabolise this fat without an increase in postprandial chylomicron concentrations. To test this hypothesis, healthy male subjects were randomised to receive, on three separate occasions, meals containing a range of doses of fat (average 8.1-19 g) and effects on postprandial lipaemia and chylomicron concentration were determined. Furthermore, to delineate the effect on lipid-rich $v$. lipid-poor (remnant) forms lipid levels were also determined in a density $<1.006 \mathrm{~g} / \mathrm{ml}$ fraction. Following consumption of the very low dose of fat the postprandial concentration of chylomicrons was unaltered, whereas following the medium dose postprandial chylomicron concentrations were significantly increased. Interestingly, this increase was only detected in the lipid-rich chylomicron fraction, with postprandial levels of chylomicron remnants remaining unchanged. In conclusion, it appears that consumption of what would be considered low to medium doses of fat are not associated with transient postprandial increases in chylomicron remnants in healthy male subjects.

Key words: Chylomicrons: apo B48: Postprandial lipid metabolism

\section{Introduction}

Impaired postprandial lipid metabolism as a risk factor for CVD is an area that has attracted great interest since the hypothesis was highlighted by Zilversmit in $1979^{(1)}$. Given that we spend the majority of our life in a postprandial state an understanding of the regulation of postprandial lipid metabolism is important. A number of studies have focused on the regulation of postprandial TAG and the results have helped establish elevated postprandial TAG concentrations as an independent risk factor for $\mathrm{CVD}^{(2)}$. Elevations in postprandial TAG concentrations result from a combination of increased concentration of TAG-rich chylomicron and VLDL particles, and their remnants. Such elevations appear to result from increased competition for lipolysis and particle clearance pathways due to the increased secretion of TAG-rich chylomicrons by enterocytes. However, postprandial remnant lipoproteins have also been established as a risk factor for atherosclerosis and CVD and it is therefore important to examine factors affecting their concentration. Following hydrolysis by endothelial lipases, TAG-depleted chylomicrons, secreted by the intestine in response to dietary fat and, thereafter, hepatically derived VLDL remnants attain a sufficiently small diameter to become entrapped within the sub-endothelial space ${ }^{(3)}$. Chylomicron and VLDL remnants contain twenty-forty

Abbreviations: LBM, lean body mass; iAUC, incremental area under the curve.

* Corresponding author: Dr Anthony James, fax +61892662958, email T.P.James@curtin.edu.au

(C) The Author(s) 2012. The online version of this article is published within an Open Access environment subject to the conditions of the Creative Commons Attribution-NonCommercial-ShareAlike licence <http://creativecommons.org/licenses/by-nc-sa/2.5/>. The written permission of 
times more cholesterol per particle compared with LDL and initiate a powerful cytokine response when retained within the arterial intima. Moreover, these remnant lipoproteins are avidly degraded by macrophages, initiating a respiratory burst and exacerbating endothelial oxidative stress ${ }^{(4,5)}$.

One of the factors that could be expected to be important in determining the extent and duration of postprandial lipaemia is the dose of lipid consumed in a test meal. Indeed, it has been reported that a stepwise increase in the postprandial serum TAG response results with increasing amount of fat consumed $^{(6,7)}$. A threshold ingestion of about $15 \mathrm{~g}$ is ordinarily required to induce transient lipaemia in otherwise healthy lean individuals. Consumption of other macronutrients, in particular carbohydrates, as well as the type of fatty acids may also confer some regulation of postprandial lipaemia, the former via insulin-regulated pathways ${ }^{(8)}$. However, due to differences in regulation of the lipolysis and clearance pathways it is possible that elevated postprandial lipaemia may not always track directly with levels of remnant lipoproteins. It is important therefore to determine the effect of dietary lipid load on arterial exposure to the post-hydrolysed pro-atherogenic remnant lipoproteins. Remnant lipoproteins, being smaller in size than their TAG-rich progenitors, are able to penetrate arterial tissue $^{(3)}$, and induce macrophage lipid loading ${ }^{(9-11)}$. The determination of remnant lipoproteins in the postprandial state is therefore of importance in evaluating the atherogenic risk of postprandial lipaemia. It is likely that accumulation of remnants of both hepatic and intestinal origin plays an important role in the initiation and progression of atherosclerosis and vascular inflammation ${ }^{(12)}$. Determination of the concentration of chylomicron particles can be done by measurement of apoB48 concentration in the circulation. More importantly, elevated apoB48 concentrations both in fasting and postprandial states have been reported in obese subjects ${ }^{(13)}$ and in type 1 and type 2 diabetics ${ }^{(14,15)}$.

Chylomicron particles appear to be constitutively synthesised and secreted by enterocytes ${ }^{(16,17)}$ even in the absence of ingested fat. Results from animal and clinical studies suggest that, unlike hepatically derived TAG-rich VLDL, basal rates of chylomicron biosynthesis are chronically modulated and not significantly influenced by singular meals containing fat. In rats, following lipid infusion there was only a $1 \cdot 5$-fold increase in the rate of chylomicron particles secreted; whereas the size of secreted lymph chylomicrons was up to $13 \cdot 8$-fold greater $^{(17)}$. Furthermore, in free-living subjects we have previously observed that the basal circulating concentration of chylomicrons represent approximately $70 \%$ of the peak levels attained throughout the day ${ }^{(18)}$. It appears therefore that the basal synthesis of chylomicrons can accommodate most of the lipid absorbed from meals by the formation and secretion of larger, lipid-rich chylomicrons. However, it remains unclear whether the extent of nascent chylomicron lipidation within the enterocyte subsequently influences plasma metabolism of chylomicrons, or thereafter interaction and kinetics within the arterial intima. Indeed, the atherogenic risk associated with postprandial lipaemia may primarily be indicative of chronic disturbances in chylomicron remnant homoeostasis, rather than reflecting single and transient meal effects. To explore this hypothesis further, healthy male subjects were randomised to receive, on three separate occasions, meals containing a range of very low to medium doses of fat (average 8.1-19 g) and effects on postprandial lipaemia and chylomicron concentration were determined.

\section{Experimental methods}

\section{Subjects}

A total of ten healthy normolipidaemic (TAG $<1.7 \mathrm{~mm}$ ) generally lean male subjects were recruited. Exclusion criteria included smoking within 2 years prior, liver or endocrine dysfunction, malabsorption syndrome, anaemia, hypothyroidism, and the use of lipid-lowering or hypertensive agents. Diabetes was excluded based on fasting serum glucose being less than $7 \mathrm{~mm}$. Informed consent was obtained from all subjects. This study was conducted according to the guidelines laid down in the Declaration of Helsinki and all procedures involving human subjects/patients were approved by the Curtin University Human Research Ethics Committee (HR 95/2007). Written informed consent was obtained from all subjects. The study is registered with the Australian New Zealand Clinical Trials Registry (ACTRN12611000795998).

\section{Design}

The study was a randomised crossover design where the subjects were required to attend three postprandial testing days. On each of these testing days the subjects were given a milkshake to consume containing either a very low, low, or medium dose of fat $(0.15,0.25$ and $0.35 \mathrm{~g} / \mathrm{kg}$ lean body mass (LBM)). The time between each of the three testing days was between 2 and 4 weeks.

\section{Diet standardisation}

As there is evidence that a subject's preceding diet affects postprandial lipaemia ${ }^{(19,20)}$ and to ensure that the subjects were in a basal metabolic state prior to consuming each of the postprandial test meals, low-fat standard meals were given to consume on the evening prior to each test day. The standardised evening meal was consumed prior to 20.00 hours after which no other food or drinks (other than water) were consumed. The foods consumed prior to their first visit were replicated exactly on all subsequent visits. These foods consisted of low-fat frozen meals, yoghurt, orange juice and muesli bars. The average composition of these meals was: energy $3273 \mathrm{~kJ}$, protein $32 \mathrm{~g}$, fat $13 \mathrm{~g}$ (saturated $6.8 \mathrm{~g}$, cholesterol $38.4 \mathrm{mg}$ ), carbohydrate $125 \mathrm{~g}$ (sugars $53.4 \mathrm{~g}$ )

\section{Determination of lean body mass}

LBM was determined using a lunar prodigy DPX-L dual-energy X-ray machine (Lunar Corp.) with Encore 2004 analysis software version $8 \cdot 50$. A whole body scan was performed once prior to commencement of the first postprandial test day. The subjects were scanned in a standard cotton gown 
and the positioning of the subjects was done according to standard procedure. In addition, prior to each scan a calibration check was done using the spine phantom provided by the manufacturer. The CV for LBM was previously determined as $0.5 \%{ }^{(21)}$.

\section{Postprandial lipoprotein assessment}

The subjects arrived at the clinical rooms for their postprandial test days at about 08.00 hours after having fasted for at least $12 \mathrm{~h}$. Upon arrival, the subject's body weight, height, waist and hip circumference were measured following the standardised procedures and using a single trained observer.

A fasting blood sample was collected after the subjects were relaxed and had sat upright for $5 \mathrm{~min}$. Following the blood sample the subjects were given their milkshake and asked to consume it within 1-2 min. To ensure that all of the milkshake was consumed, the cup was rinsed extensively with water which was then consumed. The postprandial test drink consisted of whipping cream (variable amount), chocolate flavouring $(1 \mathrm{~g})$ and water $(100 \mathrm{ml})$ only. The amount of cream was calculated for each volunteer based on their LBM (determined by dual-energy $\mathrm{X}$-ray) to ensure the fat content of the drink was $0.15,0.25$ and $0.35 \mathrm{~g} / \mathrm{kg} \mathrm{LBM}$ for the very low, low and medium test drinks, respectively. The average nutritional composition of the test meals was: very low $(8.1 \mathrm{~g}$ fat, $1.6 \mathrm{~g}$ carbohydrate and $0.4 \mathrm{~g}$ protein); low $(13.6 \mathrm{~g}$ fat, $2.0 \mathrm{~g}$ carbohydrate and $0.7 \mathrm{~g}$ protein); medium (19.0 $\mathrm{g}$ fat, $2.5 \mathrm{~g}$ carbohydrate and $1.0 \mathrm{~g}$ protein).

After consumption of the milkshake venous blood samples were collected at the following time points: 45, 90, 150 and $240 \mathrm{~min}$. Blood samples were collected in vacuum tubes containing either clotting activators for isolation of serum, EDTA for isolation of plasma and EDTA with fluoride salt for isolation of plasma for glucose determinations. Samples for serum isolation were left to clot prior to centrifugation at approximately $2000 \mathrm{~g}$ for $10 \mathrm{~min}$ at $4^{\circ} \mathrm{C}$. Aliquots of plasma and sera were collected and stored at $-80^{\circ} \mathrm{C}$ before subsequent analysis. The subjects remained mostly confined to the clinical room during the study apart from bathroom access and mostly read, or did computer-based work. The subjects were offered water and green/black tea (without milk or sugar) during the postprandial period; however, consumption times and levels were recorded and replicated on each test day.

VLDL (density $<1.006 \mathrm{~g} / \mathrm{ml}$ ) fractions were collected following ultracentrifugation of plasma samples. A density solution $(1.006 \mathrm{~g} / \mathrm{ml})$ prepared as described by Havel et al. ${ }^{(22)}$ was overlayed on $1 \mathrm{ml}$ of plasma in $5 \mathrm{ml}$ thin-walled tubes (Thermo Scientific). The samples were then spun at 40000 rpm for $21 \mathrm{~h}$ at $18^{\circ} \mathrm{C}$ using an $\mathrm{AH}-650$ swing-out rotor (Thermo Scientific). Following ultracentrifugation the lipoprotein fraction was collected by aspiration and immediately stored at $-80^{\circ} \mathrm{C}$ prior to analysis.

\section{Lipid, insulin and glucose assays}

Serum TAG, cholesterol, glucose and insulin were determined by Pathwest Laboratories using their routine automated procedures on an Architect c1600 analyser. Briefly, TAG, total cholesterol, HDL-cholesterol and glucose were determined using specific enzyme-based colorimetric reagents (Abbott Diagnostics; CV $<2 \%$ LDL-cholesterol was estimated using a modified version of the Friedewald equation ${ }^{(23)}$. Insulin was determined by Pathwest Laboratories using an Immulite 2000 Insulin Analyser (Siemens Medical Solutions Diagnostic; $\mathrm{CV}<5 \%$ ). NEFA were determined using the Wako NEFA C kit (Wako Pure Chemical Industries Ltd) according to the manufacturer's instructions.

The lipid-rich fraction was assayed directly for TAG and cholesterol using the same methods described for serum samples, their concentration was then expressed relative to that arising from $1 \mathrm{ml}$ of plasma.

\section{apoB determination}

apoB48 concentration was determined using a commercial sandwich ELISA method using a monoclonal antibody raised against the C-terminal region of apoB48 (Shibayagi Human apo B-48 ELISA Kit). For details of the method validation, refer to Kinoshita et al. ${ }^{(24)}$. Serum samples collected during the postprandial tests were analysed according to the manufacturer's instructions with the exception that samples were diluted 1 in 200 to ensure that the raised postprandial apoB48 concentrations remained within the standard curve. Determination of apoB48 in the lipid-rich fractions was performed as described for the serum samples, and was also done with a dilution of 1 in 200 prior to assay. Concentrations of apoB48 in the lipid-rich fraction were expressed relative to that arising from $1 \mathrm{ml}$ of plasma. The concentration of apoB48 in the infranatant fraction (density $>1.006 \mathrm{~g} / \mathrm{ml}$ ) was calculated by subtracting the concentration of apoB48 in the lipid-rich fraction from the total serum apo B48 concentration.

apoB concentration in the lipid-rich fraction was determined using a commercial sandwich ELISA method (Abcam apo B Human ELISA Kit) according to the manufacturer's instructions with the exception that lipoprotein fractions were diluted 1 in 1000 prior to assay. The apoB content of the fraction was then expressed relative to that arising from $1 \mathrm{ml}$ of plasma.

\section{Quantification of postprandial metabolism}

Postprandial metabolism was quantified by calculating the area under the curve (AUC) using the trapezoid method. However, in order to standardise for fasting levels, AUC comparisons between different doses of fat were corrected for the fasting level of each parameter in both the statistical comparisons and pictorially and hence represent incremental AUC (iAUC).

\section{Statistical analysis}

Analysis of the changes in baseline measures between visits was done by univariate general linear modelling, whereas changes in postprandial measures were assessed by comparing the AUC for each parameter using univariate general linear modelling with the fasting level of that parameter as a covariate for each dose of fat given. All statistics were performed using 
SPSS Statistics version 17 (SPSS Inc.). Significance was accepted at the $P<0.05$ level and data are presented as mean values with their standard errors.

\section{Results}

\section{Subject characteristics and diet}

The subject characteristics at baseline (first postprandial visit) are shown in Table 1. The subjects were on average lean (range $21 \cdot 6-25 \cdot 6 \mathrm{~kg} / \mathrm{m}^{2}$ ), normotensive, normoglycaemic and generally normolipidaemic. The subjects' weight and other anthropometric measurements were checked at each visit and did not vary significantly throughout the course of the study (data not shown), suggesting that they complied with our request to maintain their normal diet and exercise throughout the study.

\section{Postprandial TAG}

Following consumption of the very low dose of test milkshake serum TAG concentrations remained relatively unchanged with no significant difference compared with baseline (Fig. 1). Consumption of the low dose of test milkshakes resulted in a gradual increase in serum TAG concentrations, reaching a peak concentration at $150 \mathrm{~min}(P<0.05)$ before decreasing towards baseline concentrations at $240 \mathrm{~min}$ (Fig. 1). Similarly the TAG response following the medium dose of lipid gradually increased and was significantly raised at $150 \mathrm{~min}(P<0.001)$ and remained elevated at $240 \mathrm{~min}(P$ $<0 \cdot 05$, Fig. 1). Quantification of the postprandial TAG response to each dose of milkshake was determined by calculating the areas under the postprandial TAG curves (corrected for baseline levels, iAUC). The postprandial response to the medium-fat load milkshake was significantly greater $(267 \%$, $P<0 \cdot 05)$ than that observed following the very-low-fat-load. Whereas the response following the low-fat-load milkshake was not different from that observed following either the very-low- or medium-fat loads (Fig. 1).

The concentration of TAG was also determined in the lipid-rich fraction encompassing both VLDL particles and chylomicron particles (density $>1.006 \mathrm{~g} / \mathrm{ml}$ ). Following

Table 1. Baseline subject characteristics determined at the first visit (Mean values with their standard errors)

\begin{tabular}{lrr}
\hline Characteristic & Mean & SE \\
\hline Age (years) & 33.4 & 4.2 \\
Weight $(\mathrm{kg})$ & 72.1 & $2 \cdot 2$ \\
Lean mass $(\mathrm{kg})$ & 54.2 & 2.1 \\
BMI $\left(\mathrm{kg} / \mathrm{m}^{2}\right)$ & 24.0 & 0.4 \\
Waist circumference $(\mathrm{cm})$ & 84.2 & 2.1 \\
Waist:hip & 0.86 & 0.02 \\
Systolic blood pressure $(\mathrm{mmHg})$ & 120.1 & 3.9 \\
Diastolic blood pressure $(\mathrm{mmHg})$ & 74.8 & $2 \cdot 0$ \\
Total cholesterol $(\mathrm{mM})$ & 5.1 & 0.4 \\
LDL (mM) & 3.3 & 0.4 \\
HDL (mM) & 1.3 & 0.07 \\
TAG (mM) & 1.1 & 0.09 \\
Glucose & 5.1 & 0.08 \\
\hline
\end{tabular}

consumption of the very-low-fat load the TAG concentration in the lipid-rich fraction remained relatively unchanged, with no significant difference compared with the baseline concentration (Fig. 1). The TAG concentration, however, tended to increase postprandially, significantly greater or approaching significance in the later stages following the medium- and low-fat loads, respectively (Fig. 1). However, despite this the iAUC for TAG in the lipid-rich fraction was not significantly different between the fat loads (Fig. 1).

The concentration of TAG in the lipid-poor fraction (density $>1.006 \mathrm{~g} / \mathrm{ml}$ ) was calculated by subtracting the concentration of TAG in the lipid-rich fraction (density $<1.006$ $\mathrm{g} / \mathrm{ml}$ ) from the total TAG concentration in serum. The lipidpoor fraction was taken to represent a small remnant fraction encompassing lipid-depleted lipoprotein remnants of intestinal and hepatic origin. Following consumption of each fat load, however, the TAG concentration was essentially unchanged and quantification of the iAUC for TAG in this remnant fraction revealed no significant difference in response between the fat loads (Fig. 1).

\section{Postprandial apoB48}

Following consumption of both the very low and low doses of test milkshake no significant changes in serum chylomicron concentration (measured by apoB48) were observed (Fig. 2). Consumption of the medium dose of test milkshake, however, resulted in significant changes in serum chylomicron concentration, with levels of apoB48 gradually increasing, reaching a peak concentration at $150 \mathrm{~min}(P<0.01)$ before decreasing towards baseline concentrations at $240 \mathrm{~min}$ (Fig. 2). The postprandial chylomicron response as determined by the areas under the postprandial apoB48 curves (corrected for baseline levels, iAUC) varied among the different fat loads. The iAUC following the very-low-fat-dose milkshake was negative, indicating chylomicron concentration remained essentially at or below baseline concentrations for $240 \mathrm{~min}$ following the meal. A positive iAUC was observed following the low and medium doses of fat, and the response following the medium dose of fat was significantly $(P<0.05)$ greater than that observed following the very-low-dose meal. Whereas the response following the low fat dose milkshake was not different from that observed following either the very-low- or medium-fat-dose milkshakes.

The concentration of chylomicron particles in the lipid-rich fraction did not change significantly following the very low or low doses of fat load; however, it gradually increased following the medium dose of fat load, with apoB48 concentration remaining significantly higher than baseline levels at 90 and $150 \mathrm{~min}(P<0.01)$ before returning to baseline levels at 240 min (Fig. 2). Quantification of the iAUC for apoB48 in this lipid-rich fraction revealed a sequentially greater response as the dose was increased, with the iAUC following the lowand medium-dose-fat milkshakes being significantly greater (205 and $500 \%$, respectively; $P<0.05$ ) than that observed following the very-low-dose milkshake, and the iAUC following the medium-dose-fat load was greater $(97 \%, P<0.05)$ than that observed following the low-dose milkshake (Fig. 2). 
(A)

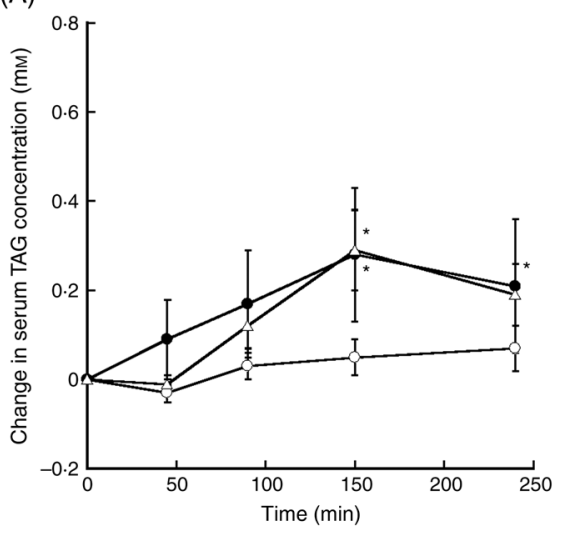

(D)

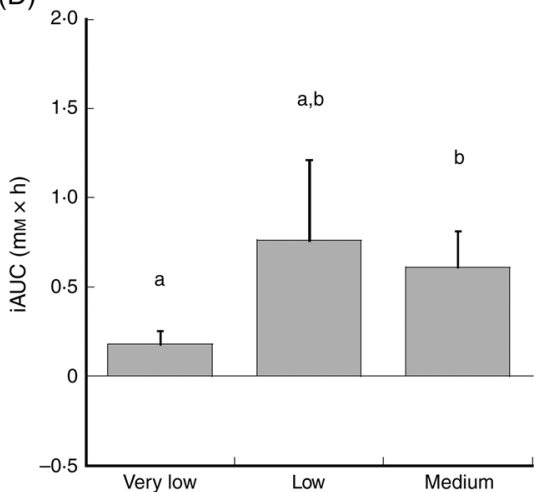

(B)

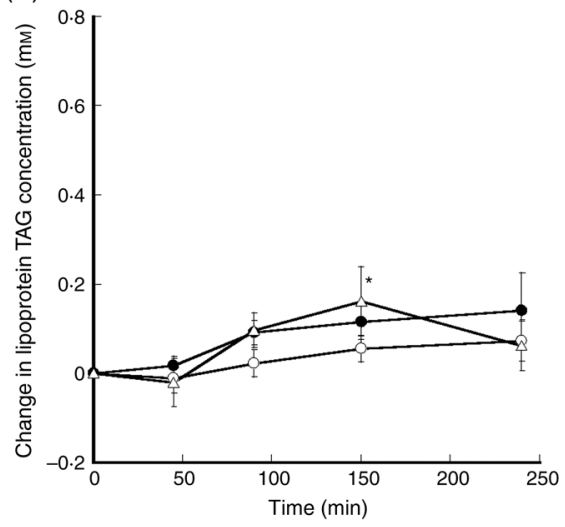

(E)

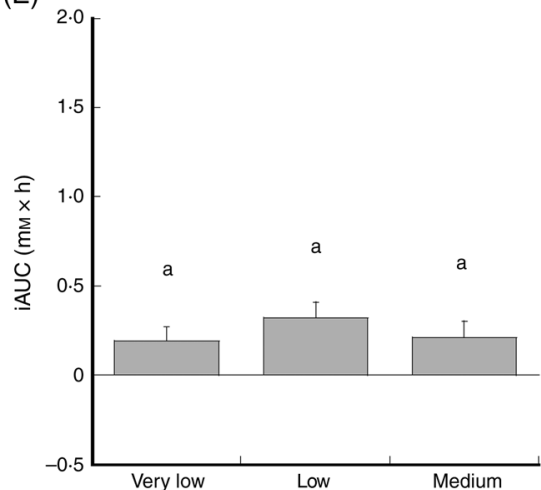

(C)

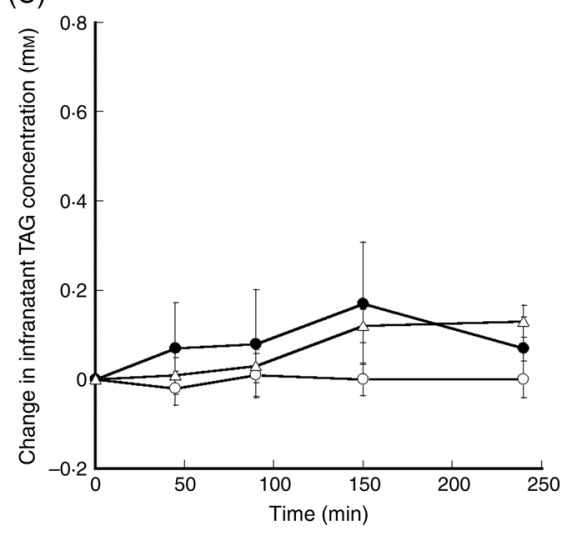

(F)

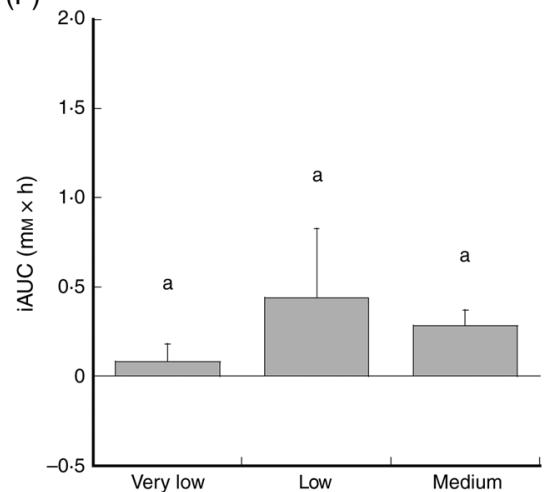

Fig. 1. Postprandial concentrations of TAG. Concentrations of TAG in serum (A), VLDL fraction (B) and infranatant (C) in the fasting state (0 min) and for 240 min after consumption of the very low $(O)$, low $(\mathbf{O})$ and medium $(\boldsymbol{\Delta})$ test meals. Quantification of the postprandial TAG response in serum (D), lipid-rich fraction (E) and lipid-poor fraction (F), shown as area under the curve AUC (corrected for baseline levels, incremental AUC (iAUC)). Values are means, with standard errors represented by vertical bars. ${ }^{*}$ Mean value was significantly different from that in the fasting state $(P<0.05)$. ${ }^{a, b}$ Mean values with unlike letters were significantly different $(P<0.05)$.

In order to estimate the effects of the fat load on postprandial small chylomicron remnant concentrations the concentration of apoB48 in the lipid-poor fraction (density $>1.006$ $\mathrm{g} / \mathrm{ml}$ ) was calculated by subtracting the concentration of apoB48 in the lipid-rich fraction (density $<1.006 \mathrm{~g} / \mathrm{ml}$ ) from the total apoB48 concentration in serum. Following consumption of each fat load, however, the apoB48 concentration was essentially unchanged and quantification of the iAUC for apoB48 in this remnant fraction revealed no significant difference in response between the fat loads.

\section{Changes in other postprandial measures}

The postprandial glucose response following all three fat loads was essentially the same, with a rapid reduction in glucose concentrations which remained suppressed up to either 90 or 150 min (Fig. 3; $P<0.05$ ) before returning to baseline levels at $240 \mathrm{~min}$ postprandially. The postprandial iAUC for glucose was not different between the fat loads (Fig. 3). The postprandial insulin response to the different fat loads was varied; the response following the very low and low doses was similar, with a general reduction in insulin levels persisting at 240 $\min$ (Fig. $3 ; P<0 \cdot 01$ ). Following the medium dose of fat insulin initially increased at $45 \mathrm{~min}(P<0.001)$ before returning, and remaining, at baseline levels (Fig. 3). As a result the postprandial iAUC for insulin following the medium dose of fat was significantly greater than that observed following the very low dose (Fig. 3; $P<0.05$ ). The postprandial NEFA response following each fat load was essentially identical, with a gradual increase in NEFA concentration postprandially reaching significantly elevated levels at $240 \mathrm{~min}$ (Fig. 3; $P<$ 0.001). Indeed there were no significant differences in the postprandial responses (iAUC) between the different fat loads (Fig. 3).

In order to assess changes in VLDL particle number the concentrations of apoB and cholesterol were determined in the lipid-rich fraction. However, there were no significant changes in these measures following or between the different fat loads. The areas under the postprandial curve for cholesterol were 0.87 (SE 0.44), 0.88 (SE 0.54) and 0.93 (SE 0.33) $\mathrm{mm} \times \mathrm{h}$ and those for apoB were 12.5 (SE 0.9), 13.5 (SE 0.9) and 12.3 (SE 0.9) $\mu \mathrm{M} \times \mathrm{h}$ following the very low, low and medium meals, respectively.

\section{Discussion}

In the present study, we examined the effects of consumption of a range of generally low doses of fat on postprandial chylomicron metabolism. Following consumption of the medium dose of fat significant increases in the circulating concentration of chylomicron particles (apoB48) were observed, whereas the very low dose did not result in any postprandial change in 
(A)

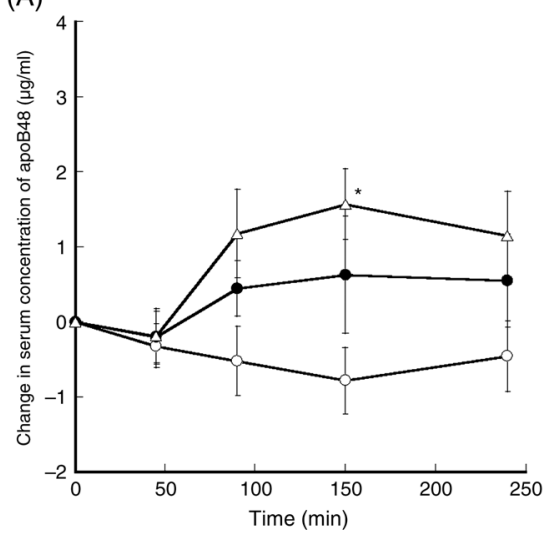

(D)

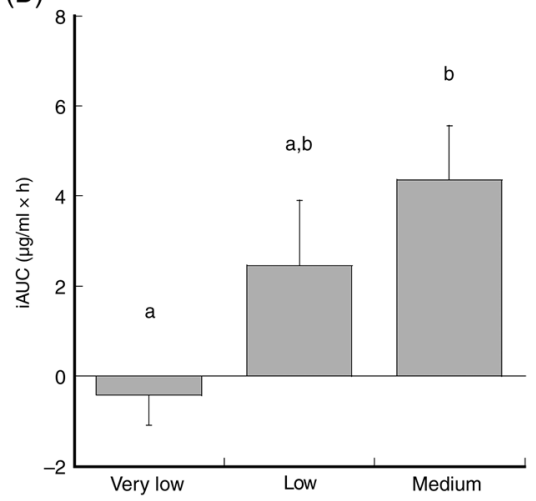

(B)

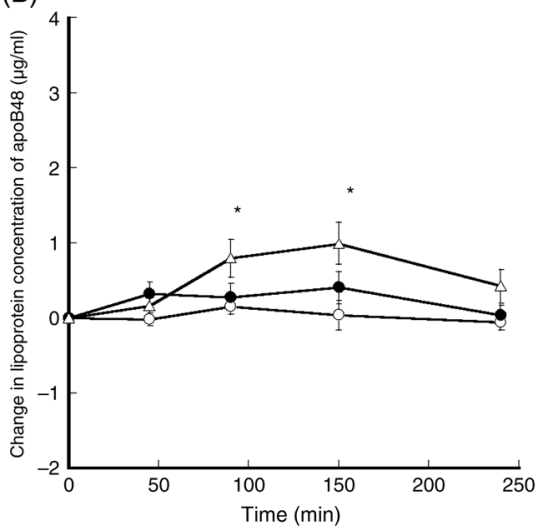

(E)

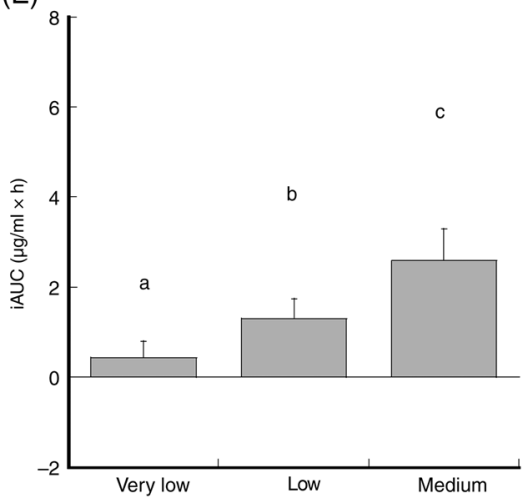

(C)

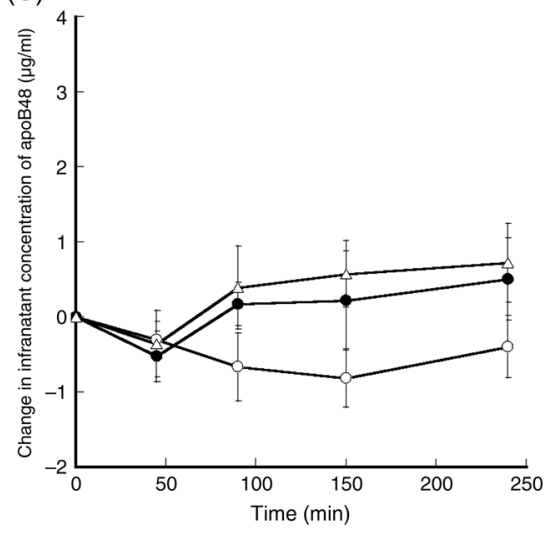

(F)

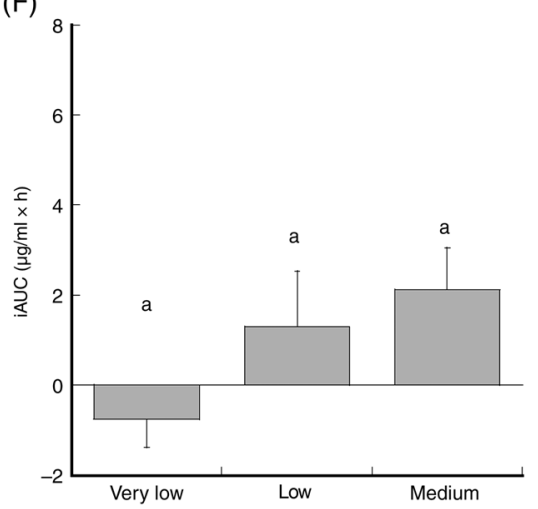

Fig. 2. Postprandial concentrations of apoB48. Concentrations of apoB48 in serum (A), lipid-rich fraction (B) and lipid-poor fraction (C) in the fasting state $(0$ min) and for 240 min after consumption of the very low $(\bigcirc)$, low $(\bigcirc)$ and medium $(\Delta)$ test meals. Quantification of postprandial apoB48 response in serum (D), lipid-rich fraction (E) and lipid-poor fraction (F), shown as area under the curve (AUC) (corrected for baseline levels; incremental AUC (iAUC)). Values are means, with standard errors represented by vertical bars. ${ }^{*}$ Mean value was significantly different from that in the fasting state $(P<0.05)$. ${ }^{a, b, c}$ Mean values with unlike letters were significantly different $(P<0.05)$.

apoB48 concentration. It appears therefore that the basal rate of chylomicron synthesis, lipolysis and clearance is sufficient to metabolise the very low doses of fat ingested without an elevation in postprandial chylomicron concentration resulting in (Fig. 2). Indeed chylomicron particles appear to be constitutively synthesised even in the absence of ingested fat, with basal (fasting) levels being approximately $70 \%$ of the peak levels attained throughout the day in free-living subjects ${ }^{(18)}$. Once secreted into the circulation chylomicrons are rapidly acted on by lipoprotein lipases prior to being cleared from the circulation with a half-life of plasma residency time between 5 and $15 \min ^{(25)}$. We also observed that consumption of the very low dose of fat did not result in a change in postprandial TAG concentrations, a finding previously reported by Dubois et al. ${ }^{(7)}$. This lack of effect on postprandial TAG concentrations again suggests that the rate of lipolysis and/or clearance capacity is sufficiently high to process these low levels of ingested fat (Fig. 1). Thus, it remains possible that the basal rate of chylomicron synthesis may accommodate low doses of ingested fat by an increase in chylomicron particle size without the need for increased chylomicron particle synthesis. However, following the consumption of the medium dose of fat it appears that an increase in chylomicron synthesis is required to accommodate the ingested fat and/or limitations in the capacity of the lipolytic, and particle clearance pathways result in a net accumulation of postprandial TAG and chylomicron particles. Interestingly, although the consumption of the low and medium doses of fat resulted in a significant increase in the postprandial TAG concentration, it appears that this effect was not localised in either the lipid-rich or lipidpoor fractions (Fig. 1).

In the circulation chylomicron particles exhibit a fairly large heterogeneity with respect to size, resulting from variations in the amount of lipid incorporation during their enterocytic formation, and the extent of lipolysis that has occurred in circulation ${ }^{(26)}$. However, in terms of atherogenic potential, it is only the lipid-depleted remnant forms that are of a size sufficiently small enough to penetrate arterial tissue ${ }^{(3)}$, and induce macrophage lipid loading ${ }^{(9-11)}$. But the quantification of chylomicron remnants remains a difficult proposal due to the heterogeneity of size. However, by determining the extent of circulating chylomicrons that are of a density $<1.006 \mathrm{~g} / \mathrm{ml}$ (lipid-rich chylomicrons), we can split the chylomicrons into those that are lipid-rich and lipid-poor, the latter of which can be used as a surrogate measure of the remnant particles which may be small enough to permit transcytotic delivery to the subendothelial space ${ }^{(3)}$. Interestingly, the effect of increasing fat load on chylomicron concentration appeared to be mostly due to an increase in lipid-rich chylomicrons, with a stepwise increase in postprandial apoB48 observed in the VLDL fraction with increasing fat load, whereas the concentration of lipid-poor chylomicrons was essentially unchanged following 
(A)

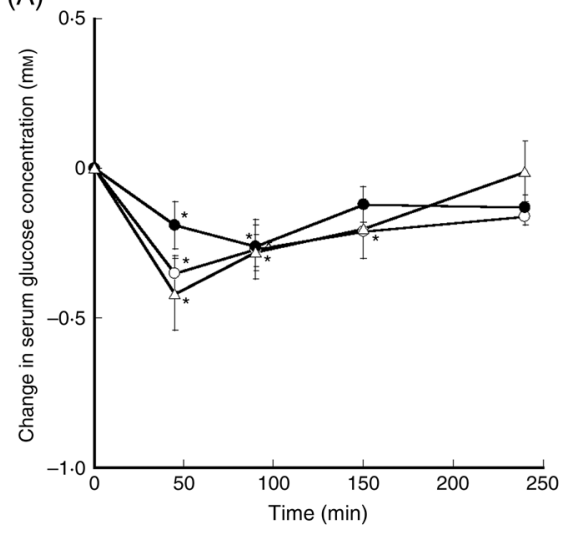

(D)

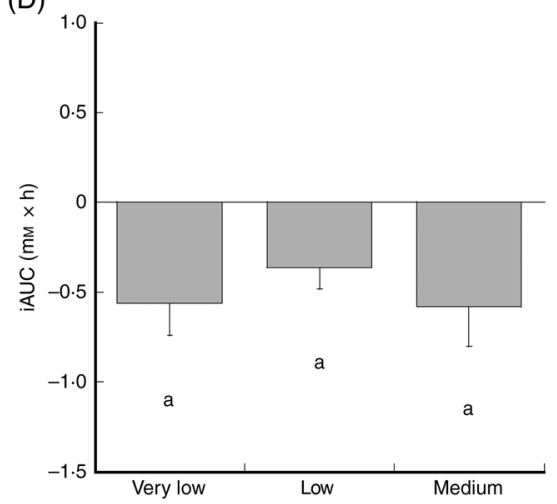

(B)

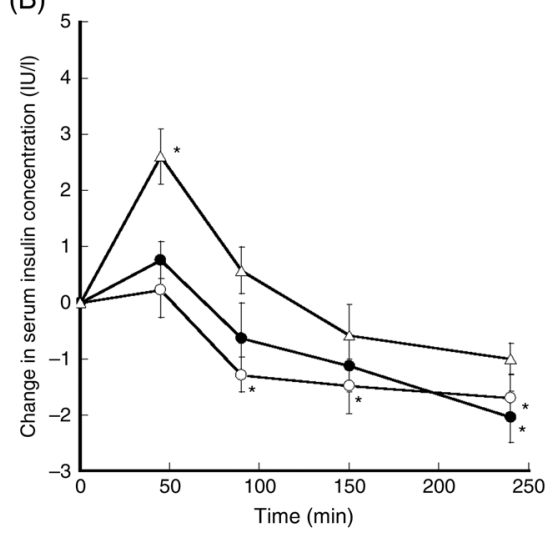

(E)

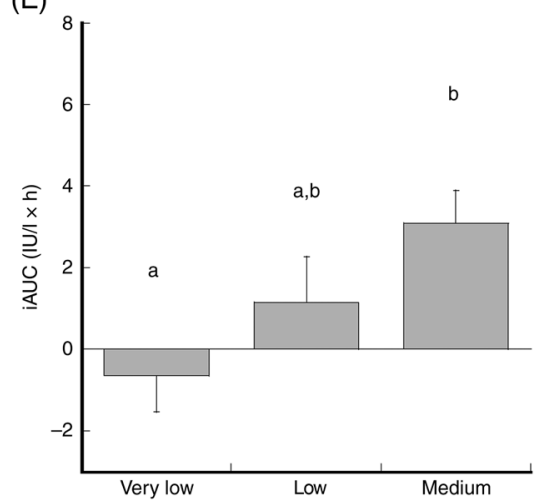

(C)

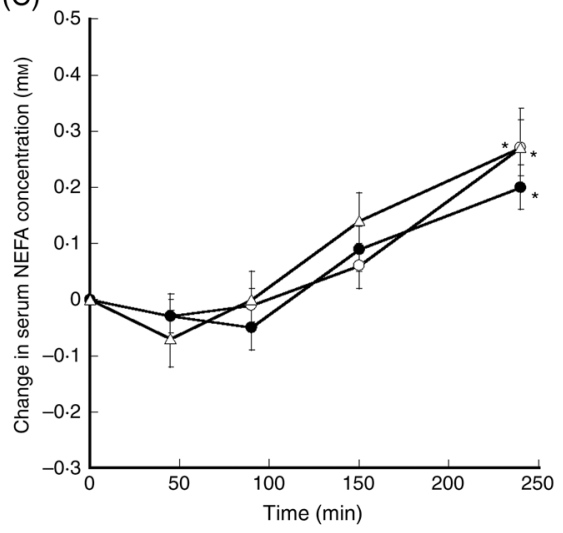

(F)

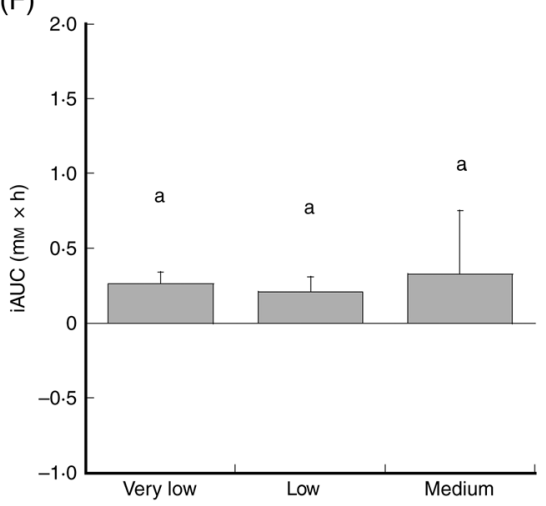

Fig. 3. Postprandial concentrations of glucose, insulin and NEFA. Serum concentrations of glucose (A), insulin (B) and NEFA (C) in the fasting state ( 0 min) and for 240 min after consumption of the very low $(O)$, low $(O)$ and medium $(\Delta)$ test meals. Quantification of postprandial responses for glucose $(D)$, insulin $(E)$ and NEFA $(F)$, shown as area under the curve (AUC) (corrected for baseline levels; incremental AUC (iAUC)). Values are means, with standard errors represented by vertical bars. ${ }^{*}$ Mean value was significantly different from that in the fasting state $(P<0.05)$. ${ }^{\text {a,b }}$ Mean values with unlike letters were significantly different $(P<0.05)$.

all doses of fat (Fig. 2). Hence, although consumption of greater doses of fat results in sequentially greater circulating chylomicron concentrations, the increase is due primarily to an increase in lipid-rich chylomicron particles. It is possible that these lipid-rich chylomicrons accumulate due to a limiting rate of lipolysis and/or an increased chylomicron secretion; however, once lipolysed the remnant forms (lipid-poor chylomicrons) appear to be rapidly cleared from circulation and do not accumulate. However, although no difference was detected in the concentration of apoB48 in the lipid-poor fraction, it is possible that greater doses of fat consumption or monitoring the postprandial response beyond $4 \mathrm{~h}$ may reveal an accumulation of lipid-poor chylomicron remnants. Interestingly, the concentration of apoB and cholesterol in the lipid-rich fraction did not change following any of the fat loads, suggesting that the VLDL particle number was unaltered. As VLDL and chylomicron particles compete for the same lipolytic pathway ${ }^{(27)}$ one may speculate that an increased secretion of lipid-rich chylomicrons may have been the primary reason for their postprandial accumulation.

The oral fat loads used in the present study consisted predominantly of lipid with only minor levels of protein and carbohydrate (maximum: $2.9 \mathrm{~g}$ carbohydrate and $1.0 \mathrm{~g}$ protein), mostly coming from the cream. The amount of added sugar (in the form of chocolate flavouring) was kept constant across the different fat loads. Consequently, protein and carbohydrate content increased slightly with increasing dose of fat load. It is likely that this is responsible for the increase in postprandial insulin concentration observed following the medium fat load. An increased postprandial insulinaemia has been reported in some ${ }^{(7,28)}$ but not all ${ }^{(29)}$ studies examining postprandial effects of increased fat load. The slight changes in insulin concentration observed in the present study in the early postprandial phase appear to have been sufficient to result in a suppression of postprandial glucose concentrations, which, however, were not sufficient to significantly reduce postprandial NEFA concentrations. Rather, NEFA concentration increased towards the end of the postprandial period, a finding that did not appear to be affected by the dose of fat load given (Fig. 3). Others have reported either a similar postprandial NEFA response or no effect of the dose of fat on the postprandial NEFA response ${ }^{(28-30)}$.

In conclusion, the findings in the present study show for the first time that consumption of low doses of fat results in no effect on the postprandial concentration of chylomicron particles. Rather, it appears that either the fat load is accommodated by the basal level of chylomicron secretion, or the rate of lipolytic, or clearance pathways are sufficient to rapidly process slight fluctuations in chylomicron concentration. Following consumption of moderate doses of fat the ensuing postprandial chylomicronaemia appears to be primarily due to an accumulation of lipid-rich chylomicron particles. Hence, in otherwise 
healthy male subjects the transient postprandial lipaemia observed following consumption of moderate doses of fat is not reflected by increased arterial exposure to pro-atherogenic (lipid-poor) chylomicron remnants. However, in subjects with remnant clearance disturbances, it is possible that acute lipid meals may result in elevations in chylomicron remnant levels. Furthermore, it remains to be determined whether consumption of doses of fat greater than those used in the present study would result in an accumulation of more lipid-poor remnant chylomicron particles. The findings from the present study raise the possibility that there is merit in limiting the amount of fat consumed during mealtimes in order to reduce the circulating concentration of pro-atherogenic (lipid-poor) chylomicron remnants.

\section{Acknowledgements}

A. P. J. conceived the idea of the study, developed the study design, conducted the study, analysed the data and wrote the manuscript. J. C. M. developed the study design, analysed the data and wrote the manuscript. The authors are grateful for the technical assistance provided by Camille Gesquiere and Charlotte Michel. This work was supported by both the Australian Technology Network Centre for Metabolic Fitness and Curtin University Office of Research \& Development via small grant funds.

The authors declare no conflict of interest.

\section{References}

1. Zilversmit DB (1979) Atherogenesis: a postprandial phenomenon. Circulation 60, 473-485.

2. Jackson KG, Poppitt SD \& Minihane AM (2012) Postprandial lipemia and cardiovascular disease risk: interrelationships between dietary, physiological and genetic determinants. Atherosclerosis 220, 22-33.

3. Mamo JC \& Wheeler JR (1994) Chylomicrons or their remnants penetrate rabbit thoracic aorta as efficiently as do smaller macromolecules, including low-density lipoprotein, high-density lipoprotein, and albumin. Coron Artery Dis 5, 695-705.

4. Bentley C, Hathaway N, Widdows J, et al. (2011) Influence of chylomicron remnants on human monocyte activation in vitro. Nutr Metab Cardiovasc Dis 21, 871-878.

5. Wilhelm MG \& Cooper AD (2003) Induction of atherosclerosis by human chylomicron remnants: a hypothesis. J Atheroscler Thromb $\mathbf{1 0}$, 132-139.

6. Cohen JC, Noakes TD \& Benade AJ (1988) Serum triglyceride responses to fatty meals: effects of meal fat content. Am J Clin Nutr 47, 825-827.

7. Dubois C, Beaumier G, Juhel C, et al. (1998) Effects of graded amounts $(0-50 \mathrm{~g})$ of dietary fat on postprandial lipemia and lipoproteins in normolipidemic adults. Am J Clin Nutr 67, 31-38.

8. Lopez-Miranda J, Williams C \& Lairon D (2007) Dietary, physiological, genetic and pathological influences on postprandial lipid metabolism. Br J Nutr 98, 458.

9. Batt KV, Avella M, Moore EH, et al. (2004) Differential effects of low-density lipoprotein and chylomicron remnants on lipid accumulation in human macrophages. Exp Biol Med 229, 528-537.

10. Tomono S, Kawazu S, Kato N, et al. (1994) Uptake of remnant like particles (RLP) in diabetic patients from mouse peritoneal macrophages. I Atheroscler Thromb 1, 98-102.
11. Yu KC \& Mamo JC (1997) Regulation of cholesterol synthesis and esterification in primary cultures of macrophages following uptake of chylomicron remnants. Biochem Mol Biol Int 41, 33-39.

12. Schwartz EA \& Reaven PD (2012) Lipolysis of triglyceride-rich lipoproteins, vascular inflammation, and atherosclerosis. Biochim Biophys Acta 1821, 858-866.

13. Mamo JC, Watts GF, Barrett PH, et al. (2001) Postprandial dyslipidemia in men with visceral obesity: an effect of reduced LDL receptor expression? Am J Physiol Endocrinol Metab 281, E626-E632.

14. Curtin A, Deegan P, Owens D, et al. (1996) Elevated triglyceride-rich lipoproteins in diabetes. A study of apolipoprotein B-48. Acta Diabet 33, 205-210.

15. Mangat R, Su JW, Lambert JE, et al. (2011) Increased risk of cardiovascular disease in Type 1 diabetes: arterial exposure to remnant lipoproteins leads to enhanced deposition of cholesterol and binding to glycated extracellular matrix proteoglycans. Diabet Med 28, 61-72.

16. Hayashi H, Fujimoto K, Cardelli JA, et al. (1990) Fat feeding increases size, but not number, of chylomicrons produced by small intestine. Am J Physiol 259, G709-G719.

17. Martins IJ, Sainsbury AJ, Mamo JC, et al. (1994) Lipid and apolipoprotein B48 transport in mesenteric lymph and the effect of hyperphagia on the clearance of chylomicron-like emulsions in insulin-deficient rats. Diabetologia 37, 238-246.

18. James AP, Slivkoff-Clark K \& Mamo JC (2003) New insights into cardiovascular disease risk in subjects with visceral obesity. Asia Pac J Public Health 15, Suppl., S37-S40.

19. Karpe F (1997) Effects of diet on postprandial lipaemia - a suggestion for methodological standardization. Nutr Metab Cardiovasc Dis 7, 44-55.

20. Slivkoff-Clark K, James AP, Kerr D, et al. (2004) The effect of diet standardisation on postprandial chylomicron response. Asia Pac J Clin Nutr 13, S69.

21. Whiteford J, Ackland TR, Dhaliwal SS, et al. (2010) Effects of a 1 -year randomized controlled trial of resistance training on lower limb bone and muscle structure and function in older men. Osteoporos Int 21, 1529-1536.

22. Havel RJ, Eder HA \& Bragdon JH (1955) The distribution and chemical composition of ultracentrifugally separated lipoproteins in human serum. J Clin Invest 34, 1345-1353.

23. Bairaktari E, Hatzidimou K, Tzallas C, et al. (2000) Estimation of LDL cholesterol based on the Friedewald formula and on apo B levels. Clin Biochem 33, 549-555.

24. Kinoshita M, Kojima M, Matsushima T, et al. (2005) Determination of apolipoprotein B-48 in serum by a sandwich ELISA. Clin Chim Acta 351, 115-120.

25. Park Y, Damron BD, Miles JM, et al. (2001) Measurement of human chylomicron triglyceride clearance with a labeled commercial lipid emulsion. Lipids 36, 115-120.

26. Cohn JS, Marcoux C \& Davignon J (1999) Detection, quantification, and characterization of potentially atherogenic triglyceriderich remnant lipoproteins. Arterioscler Thromb Vasc Biol 19, 2474 2486.

27. Karpe F, Bickerton AS, Hodson L, et al. (2007) Removal of triacylglycerols from chylomicrons and VLDL by capillary beds: the basis of lipoprotein remnant formation. Biochem Soc Trans 35, 472-476.

28. Murphy MC, Isherwood SG, Sethi S, et al. (1995) Postprandial lipid and hormone responses to meals of varying fat contents: modulatory role of lipoprotein lipase? Eur J Clin Nutr 49, 578-588.

29. Shishehbor F, Roche HM \& Gibney MJ (1999) The effect of low and moderate fat intakes on the postprandial lipaemic and hormonal responses in healthy volunteers. BrJ Nutr 81, 25-30.

30. Gibney MJ \& Daly E (1994) The incorporation of $n$-3 polyunsaturated fatty acids into plasma lipid and lipoprotein fractions in the postprandial phase in healthy volunteers. Eur J Clin Nutr 48, 866-872. 